\title{
Diacronie
}

Studi di Storia Contemporanea

$\mathrm{N}^{\circ} 20,4 \mid 2014$

II diritto militante

\section{Esclusione sociale e violenza istituzionale}

Il tema della salute mentale in «Quale giustizia»

Francesco Mantovani

\section{(2) OpenEdition \\ Journals}

\section{Edizione digitale}

URL: http://journals.openedition.org/diacronie/1695

DOI: 10.4000/diacronie. 1695

ISSN: 2038-0925

Editore

Association culturelle Diacronie

Notizia bibliografica digitale

Francesco Mantovani, «Esclusione sociale e violenza istituzionale », Diacronie [Online], N²0, 4 | 2014, documento 6, Messo online il 01 décembre 2014, consultato il 20 avril 2019. URL : http://

journals.openedition.org/diacronie/1695; DOI : 10.4000/diacronie.1695 


\section{Diacronie}

N. 20 | 4|2014 Il diritto militante

\section{6/}

\section{Esclusione sociale e violenza istituzionale Il tema della salute mentale in «Quale giustizia»}

Francesco MANTOVANI *

Il presente saggio è incentrato sul rapporto tra salute mentale e diritto nel contesto riformistico italiano degli anni Settanta del Novecento. La lotta condotta insieme dagli esponenti di Magistratura Democratica e Psichiatria Democratica per la chiusura degli ospedali psichiatrici e dei manicomi giudiziari è ripercorsa attraverso la lettura di «Quale giustizia», rivista giuridica impegnata in questa $e$ in altre campagne: in essa sono presenti da un lato articoli di psichiatri e giuristi, dall'altro alcune cronache giudiziarie significative. In questa sede si vuole mettere in luce l'efficacia e i limiti concreti dell'impiego del «diritto come arma» per portare avanti riforme giuridiche e sociali, attraverso l'appello alla Costituzione.

\section{Introduzione. Psichiatria e diritto}

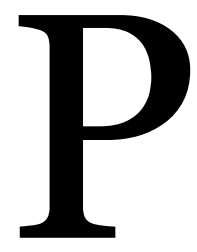

uò la contestazione servirsi del diritto per raggiungere i propri obiettivi? Certamente la legge è stata in molti casi uno strumento con cui l'autorità ha regolato e delimitato le attività dei singoli e della società: secondo la tradizionale lettura marxista, la dominazione sociale troverebbe proprio nel diritto la legittimazione più solida, dato che la sua conoscenza e la sua manipolazione sono limitate a pochi esperti. Tuttavia, negli ultimi decenni la storia e la sociologia si sono sempre più interessate ai casi in cui il diritto fu rivolto contro lo stato, al punto da diventare uno stimolo alla mobilitazione civile, dal momento che, oltre a essere l'insieme delle norme giuridiche, esso incarna i valori culturali e simbolici della società. Riprendendo una felice espressione della studiosa francese Liora Israël, il diritto può essere quindi letto come un' «arma a doppio taglio» e lo studio da parte delle 
scienze umane del suo impiego nella contestazione ne ha mostrato un'immagine meno monolitica ${ }^{1}$. In questo contesto, fondamentali sono state le ricerche incentrate sull'attività di chi si è servito del diritto come arma di attacco o di difesa: i magistrati e gli avvocati. Quello che in questa sede si vuole analizzare è il contributo che la magistratura politicamente impegnata ha dato nella campagna per la chiusura degli ospedali psichiatrici in Italia. Non solo: fu proprio l'associazionismo di giudici e avvocati a fornire un modello per gli altri settori professionali, tra cui quello psichiatrico.

Il contesto storico in cui ciò avvenne, ovvero gli anni che vanno dal 1968 al 1978, furono caratterizzati da una straordinaria concentrazione di riforme: l'intera società italiana fu infatti attraversata da grandi cambiamenti istituzionali, sociali, economici e culturali. Vennero per esempio approvati lo Statuto dei lavoratori (l. 20 maggio 1970, n. 300), la legge sul divorzio (l. 1 dicembre 1970, n. 898), la riforma del sistema carcerario (l. 26 luglio 1975, n. 354), la legalizzazione dell'aborto (l. 22 maggio 1978, n. 194) e molte altre. Non si trattò di un'operazione indolore: il dibattito tra le forze più conservatrici e quelle progressiste fu molto acceso e non a caso gli anni Settanta furono segnati anche da stragi e attentati compiuti dai gruppi extraparlamentari.

Oltre ai movimenti giovanili e operai, a partire dagli anni Sessanta larghi segmenti delle professioni mediche, tecniche e legali furono coinvolte nella critica alla società e alle istituzioni. È in questa fase che emerse la figura del professionista impegnato definito «intellettuale specifico»² da Michel Foucault in ambito francese - agiva sul sociale a partire dalle proprie conoscenze settoriali. Il primo passo compiuto da molti di costoro fu uscire dall'autoreferenzialità, dal tecnicismo e dal conformismo che caratterizzava le rispettive professioni. Ciò che accomunò le varie esperienze di riforma fu quindi l'utilizzo dei singoli saperi come strumento di lotta: durante il decennio in questione, si assistette infatti a una politicizzazione di mestieri che fino ad allora erano stati solo marginalmente coinvolti nel dibattito istituzionale. Fu l'agire concreto all'interno del proprio impiego con finalità di trasformazione a dare carattere politico all'impegno ${ }^{3}$. Vennero messe in discussione le forme di associazionismo già esistenti e presto ne nacquero di nuove, con l'intento di riformare e svecchiare le professioni dal proprio interno: fecero da apripista Magistratura democratica e la Federazione dei sindacati degli avvocati e procuratori italiani (FESAPI), nate entrambe nel 1964,

\footnotetext{
${ }^{1}$ ISRAËL, Liora, Le armi del diritto, Milano, Giuffrè, 2012, p. 9.

2 MALATESTA, Maria, Professioni e impegno negli anni Sessanta agli anni Ottanta, in MALATESTA, Maria (a cura di), Impegno e potere. Le professioni italiane dall'Ottocento a oggi, Bologna, Bononia University press, 2011, p. 73

3 Ibidem, pp. 74-75.
} 
ispiratrici delle altre associazioni di settore, come Psichiatria democratica e Medicina democratica.

Una delle questioni su cui si concentrò maggiormente il dibattito fu quella dell'istituzione manicomiale. Nel corso degli anni Sessanta, l'esperienza della «comunità terapeutica» avviata presso l'Ospedale Psichiatrico di Gorizia da Franco Basaglia mise in luce le contraddizioni interne a queste strutture, luoghi di esclusione e sevizie invece che di cure: a Gorizia venne messa in pratica una nuova metodologia di cura dei malati di mente, poi descritta nel testo L’istituzione negata (1968).

Senza negare la realtà dell'infermità mentale, ciò che viene messo in luce è la violenza istituzionale che regola i rapporti tra la società e i malati. Si tratta di una lettura non limitata all'ambito specifico della psichiatria, dal momento che i manicomi vengono inseriti in quelle che Basaglia chiama «le istituzioni della violenza», nelle quali la violenza è giustificata da una «colpa» (il carcere) o da una «malattia» (l'ospedale psichiatrico). Compito dei tecnici a cui sono appaltati questi istituti è «adattare gli individui ad accettare la loro condizione di "oggetti di violenza"»4, dando per scontato che questa sia l'unica realtà loro concessa. In questo modo, il malato di mente non è altro che un «uomo senza diritti, soggetto al potere dell'istituto, quindi alla mercé dei delegati (i medici) della società, che lo ha allontanato ed escluso»5. Risulta evidente quindi che il fine degli ospedali psichiatrici non sia tanto la cura e - possibilmente - il reinserimento dei pazienti nella società, bensì la loro custodia in un ambiente separato e la tutela dei "sani" nei confronti della follia.

Attraverso il «rovesciamento» di questa istituzione coercitiva, quello che Basaglia e l'équipe di medici, infermieri e collaboratori fecero a Gorizia fu rifiutare questo tipo di «mandato sociale». Ciò comportò la costruzione di un rapporto diverso con i pazienti, mostrando attraverso la loro responsabilizzazione che un'alternativa al manicomio tradizionale era praticabile ${ }^{6}$. Uno degli aspetti caratteristici di questo discorso era l'oscillazione e l'apparente contraddizione che poteva instaurarsi fra il bisogno di autorità (necessaria per condurre la trasformazione del sistema) e il bisogno di conquistare la propria libertà che passava attraverso la presa di responsabilità, sia da parte del personale curante che da parte dei malati. Tuttavia è evidente che il movimento comune, compiuto attraverso la negazione della propria faccia istituzionale (tanto per i malati quanto per i medici e gli infermieri), portasse alla negazione non solo dell'istituzione in questione, bensì di ogni sistema di oppressione e di sopruso.

\footnotetext{
4 BASAGLIA, Franco (a cura di), L’istituzione negata, Torino, Einaudi, 1968, p. 116.

5 Ibidem, pp. 122-123.

${ }^{6}$ Ibidem, p. 329.
} 
Nella pratica, lo svolgimento delle attività della «comunità terapeutica» era regolato dalle riunioni, le quali erano ovviamente affiancate dal programma ospedaliero tradizionale (visita dei sanitari ai reparti, pasti, terapie, ecc.). Lo scopo delle numerosissime assemblee era far sì che i degenti si riappropriassero della loro personalità attraverso le «scelte» e attraverso la discussione condotta alla pari con il personale medico. «Mettere tra parentesi» la malattia mentale mostrò che tutto ciò fosse più un prodotto che un dato di fatto, mettendo così in discussione le fondamenta del sistema su cui si reggeva l’istituzione manicomiale italiana. L’istituzione negata ebbe una grande diffusione, tanto da divenire durante il Sessantotto uno dei testi guida della cultura anti-autoritaria giovanile.

All'interno della professione psichiatrica si venne perciò a consolidare un forte impegno, inteso da un lato come critica della società e del «nesso sapere/potere» che la regolava, dall'altro come ricerca di un'attività rispettosa dei diritti e della soggettività dei pazienti7. Quello che Basaglia e collaboratori vollero fare non fu solo una riforma dei metodi terapeutici, bensì una messa in discussione del sistema che vi stava dietro, sul quale si reggevano i manicomi, «uscendo dal suo campo specifico e tentando di agire sulle contraddizioni sociali» ${ }^{8}$. Questi spunti critici furono accolti dal gruppo di magistrati riformisti e la battaglia che portò nel 1978 all'approvazione della legge n. 180 fu condotta anche sul piano costituzionale: a essere messa in discussione fu la legittimità della legge del 14 febbraio 1904, n. 36, che - salvo alcune modifiche regolava ancora l'istituzione manicomiale italiana.

Il tema della salute mentale e della riforma degli ospedali psichiatrici viene affrontato in questa sede a partire dalla lettura delle fonti presenti nella rivista «Quale giustizia», dalle quali emerge un utilizzo del diritto come strumento per portare avanti campagne riformiste e per sensibilizzare l'opinione pubblica, vista la vasta risonanza che la questione ebbe in Italia.

\section{Magistratura democratica e Psichiatria democratica}

Tra gli anni Cinquanta e Sessanta si assistette alla progressiva messa in pratica delle cosiddette «norme programmatiche» della Costituzione repubblicana, inizialmente rimandate per il principio di continuità dell'ordinamento: infatti, salvo la parte abrogata già dal 1944, la legislazione in vigore era quella emanata sotto il fascismo. Nel corso degli anni il tema del rispetto delle norme costituzionali divenne il motore di

7 MALATESTA, Maria (a cura di), op. cit., p. 79.

8 BASAGLIA, Franco (a cura di), op. cit., p. 8. 
molte delle lotte condotte sul terreno giuridico da avvocati e giudici, che avevano riscoperto forme associative abolite durante il fascismo: nel 1944 era stata infatti rifondata l'Associazione nazionale magistrati (ANM).

Nel corso degli anni Sessanta, il benessere economico era diffuso ormai in settori sempre più ampi della società italiana, la quale però si trovò sempre più limitata in un ordinamento non più al passo coi tempi: il decennio fu inaugurato infatti con la prima grande stagione degli scioperi, sintomo di questa insofferenza. Spinte di questo genere verso il rinnovamento ebbero ripercussioni anche nelle istituzioni e nella magistratura: da un lato vi fu la fase dei governi di centro-sinistra, dall'altro con la legge Breganze (l. 25 luglio 1966, n. 570) fu abolito l'obbligo del concorso per la progressione di carriera dei magistrati di tribunale a giudici d'appello. Eliminati i concorsi interni, i magistrati si sentirono più liberi di seguire i propri orientamenti, senza preoccupazioni di carriera9. Fu proprio all'inizio degli anni Sessanta che vennero a costituirsi alcune correnti interne all'ANM: tra il 1962 e il 1963 nacque Magistratura indipendente, rappresentante la componente moderata, mentre nel luglio 1964 fu costituita a Bologna Magistratura democratica. Fin dall'inizio i giudici iscritti si batterono per la piena applicazione dei principi costituzionali, con l'intento di contribuire alla trasformazione della società in senso emancipatorio ed egualitario, promuovendo una maggiore giustizia sociale.

Durante il triennio 1968-1970, l'Italia conobbe una notevole accelerazione della spinta sociale al cambiamento, anche se da parte politica non si riuscì sempre a rispondere adeguatamente al tali richieste. In questo contesto Magistratura democratica si interrogò sul ruolo che i giudici dovessero svolgere: non bastava essere interpreti della legge e promuovere la democratizzazione dell'esercizio della funzione giudiziaria, bisognava farsi garanti dei diritti della classi lavoratrici e dei ceti sottoposti. Secondo il magistrato Giuseppe Borrè, l'aspetto centrale che caratterizzò questo cambio di rotta fu la demistificazione, cioè «la rottura di miti antichi, autorevoli, mai posti in dubbio. [...] Occorreva consumare uno scisma entro la cittadella della giurisdizione» ${ }^{10}$. Questo portò alla definizione di una linea politico-culturale che privilegiava il cosiddetto «"intervento esterno", cioè il rapporto e la collaborazione con le forze

\footnotetext{
9 BERTI ARNOVALDI VELI, Giuliano, Post-fazione: il Sessantotto e il mondo del diritto in Italia, in ISRAËL, Liora, op. cit., p. 115 .

10 PEPINO, Livio, «Appunti per una storia di Magistratura democratica», in Questione giustizia, 1, 2002, pp. 11-12, URL:

< http://www.magistraturademocratica.it/mdem/materiale/storia_md.pdf > [consultato il 6 novembre 2014].
} 
politiche e sociali che operano per il cambiamento ${ }^{11}$. La scelta di assumere posizioni che possono essere definite più marcatamente "di sinistra" accentuò le divisioni interne: nel 1969 Magistratura democratica subì una scissione interna guidata da uno dei suoi fondatori, Adolfo Beria d'Argentine. In ogni caso, l'associazione continuò le proprie attività indirizzandole all'applicazione dello Statuto dei lavoratori e rifacendosi direttamente all'articolo 3 della Costituzione, che impegna la Repubblica a «rimuovere gli ostacoli di ordine economico e sociale, che, limitando di fatto la libertà e l'eguaglianza dei cittadini, impediscono il pieno sviluppo della persona umana e l'effettiva partecipazione di tutti i lavoratori all'organizzazione politica, economica e sociale del Paese».

Proprio all'esempio di Magistratura democratica si ispirò Franco Basaglia, che nel 1971 era stato nominato direttore dell'Ospedale Psichiatrico di Trieste. Qui riorganizzò i servizi psichiatrici secondo le disposizioni della cosiddetta Legge Mariotti (1. 12 febbraio 1968, n. 132), la quale prevedeva la riorganizzazione complessiva degli ospedali, trasformati ora in enti pubblici. Si trattò di una riforma importante, dal momento che introdusse una serie di garanzie all'interno dei manicomi, ma d'altra parte essa non intaccò la legislazione di epoca giolittiana che regolava il loro funzionamento. È su tale questione che le battaglie per l'emancipazione e la liberazione condotte dagli psichiatri basagliani si intrecciarono a quelle di Magistratura democratica. Dopo una fase in cui i medici preferirono adottare forme d’impegno più «fluide», all'inizio degli anni Settanta essi cercarono di ridefinire e consolidare la propria identità attraverso la fondazione di associazioni sul modello di Magistratura democratica. Nella conferenza stampa del 13 ottobre 1973 fu presentato il documento programmatico dell'appena nata Psichiatria democratica, costituitasi a Bologna l'8 settembre precedente. Il gruppo fondatore comprendeva Franca Ongaro Basaglia, Franco Basaglia, Domenico Casagrande, Franco Di Cecco, Tullio Fragiacomo, Vieri Marzi, Piera Piatti, Agostino Pirella, Michele Risso, Lucio Schittar, Antonio Slavich ${ }^{12}$. Primo segretario fu Gian Franco Minguzzi, professore presso l'Università di Bologna, il quale ricoprì tale carica fino al 1977, quando la sua linea entrò in conflitto con altre correnti dell'associazione. Da parte loro, i magistrati democratici si dichiararono disposti a collaborare «per la ristrutturazione delle finalità comuni che si riassumono, in definitiva, nella finalità di garantire in ogni caso il rispetto della Costituzione» ${ }^{13}$.

\footnotetext{
11 Storia $d i \quad$ MD, democratica, URL: <http://magistraturademocratica.it/mdem/storia.php> [consultato il 6 novembre 2014]. 12 MALATESTA, Maria (a cura di), op . cit., pp. 80-82; BABINI, Valeria P., Liberi tutti. Manicomi e psichiatri in Italia: una storia del Novecento, Bologna, Il Mulino, 2011, p. 285.

13 «Psichiatria democratica», in Quale giustizia, 21-22, 1973, p. 583.
} 


\section{3. «Quale giustizia» e la questione manicomiale}

Il richiamo alla messa in pratica dei principi costituzionali è stata allo stesso tempo la fonte da cui è nata e lo stimolo con cui è andata avanti l'esperienza di «Quale giustizia», rivista di Magistratura democratica pubblicata dal 1970 al 1979: è probabile che non sia un caso che l'arco temporale coperto da questa pubblicazione coincida pressoché interamente con la già citata età delle riforme ${ }^{14}$. Fondata a Bologna, al suo interno collaborarono magistrati e giovani ricercatori universitari di giurisprudenza, tra cui Vincenzo Accattatis, Pasquale Basile, Luigi De Marco, Marco Ramat, Salvatore Senese, Domenico Pulitanò ed Elisa Ceccarelli. L'obiettivo del progetto era fornire un valido strumento di «documentazione, critica e di ricerca» sulla realtà italiana, non solo agli esperti in materia: tendendo presente una «prospettiva ben definita», i giudici e gli altri collaboratori del periodico contribuirono con articoli incentrati sulle tematiche più disparate, tra cui la difesa del lavoro e della salute, il pericolo delle violenze compiute da movimenti neofascisti, la repressione poliziesca e giudiziaria della rivolta studentesca, il diritto di famiglia e la questione manicomiale. Il richiamo alla Costituzione e ai suoi valori, il «solo padrone» riconosciuto da questi magistrati, comportò quindi il rifiuto di una giustizia repressiva intesa come il «braccio secolare del "sistema" », oltre alla necessaria ricerca di un'alternativa che potesse promuovere una maggiore giustizia sociale ${ }^{15}$.

Tra le tematiche a cui fu data maggiore importanza nei numeri di «Quale giustizia», alla questione dei manicomi e della salute mentale spetta un posto di spicco. Lo dimostra la frequenza con cui essa compare tra il 1972 fino al 1977, in forma di cronaca giudiziaria, focus o articolo; nel numero 17-18 del 1972, per esempio, ci si concentra

\footnotetext{
14 Un'altra interessante pubblicazione del periodo fu La Questione criminale, curata dalla scuola penalistica di Bologna che faceva capo a Franco Bricola, docente di diritto penale presso l'Alma mater studiorum.

15 RAMAT, Marco, «Un solo padrone», in Quale giustizia, 1, 1970, p. 7. In questo editoriale è espresso chiaramente cosa intendessero per giustizia i redattori della rivista: «Ci torna in mente questo bellissimo e amaro ritratto fatto da A. France: "Ho conosciuto un giudice austero. Si chiamava Thomas de Maulon ed apparteneva alla piccola nobiltà provinciale. Era entrato volontariamente nella magistratura sotto il settennato del maresciallo McMahon nella speranza di rendere un giorno la giustizia in nome del Re. Aveva dei princìpi che poteva credere irremovibili, non avendoli mai mossi. Quando si muove un principio, si trova sempre qualcosa sotto, e ci si accorge che non era un principio. Thomas de Maulon teneva accuratamente al riparo della sua curiosità i propri princìpi religiosi e sociali". Ecco perché abbiamo dichiarato 'eretica' questa rivista. Il significato etimologico di eresia è 'ricerca', 'scelta'. Ma per cercare e per scegliere è necessario lo stimolo della curiosità, il coraggio di essere curiosi. "Aveva dei princìpi che poteva credere irremovibili, non avendoli mai mossi": a quanti uomini, a quanti magistrati si può attribuire questo stato d' animo?».
} 
soprattutto sul manicomio giudiziario, mentre il numero 29 del 1974 tratta ampiamente il problema delle situazioni giuridiche sospese. Più in generale, i redattori della rivista diedero molto spazio al complesso rapporto tra la giustizia e la salute, viste le sue ampie ricadute sociali.

La battaglia per la chiusura degli istituti psichiatrici fu condotta su «Quale giustizia» principalmente attraverso due vie. Da un lato si mostrò quanto la legge che regolava il funzionamento degli ospedali psichiatrici e dei manicomi giudiziari avesse come obiettivo l'esclusione sociale dei pazienti, non la loro cura; di conseguenza, i ricoveri di persone non malate, i soprusi e le terapie punitive e disumane, che venivano a galla quando un fatto di cronaca diventava di pubblico dominio, altro non erano se non una forma di «violenza legalizzata» facente parte di un vasto sistema coercitivo ${ }^{16}$. D’altra parte, fu proprio il diritto a fornire il principale strumento di contestazione: l'appello alla Costituzione per la definitiva chiusura dei manicomi è presente negli articoli della rivista, con intensità sempre maggiore dal 1974, cioè quando fu avanzata la questione di illegittimità costituzionale della legge che regolava le situazioni giuridiche sospese in caso di infermità mentale sopravvenuta all'imputato (dell'artt. 148 c.p. e 88 c.p.p.), in contrasto col diritto alla difesa espresso nell'art. 24, $2^{\circ}$ Comma, Cost., che prevede a ogni grado della procedura penale la partecipazione dell'interessato e la sua assistenza per mezzo di un difensore ${ }^{17}$. Si trattava ovviamente solo di una delle tante contraddizioni sulle quali era costruito il sistema manicomiale.

\subsection{La critica della legislazione dei manicomi}

Tutto il sistema che regolava la cura della malattia mentale, dal ricovero al funzionamento degli istituti psichiatrici, era legata alla legge 14 febbraio 1904, n. 36 e al relativo regolamento di esecuzione del 1909, che dettavano le «disposizioni sui manicomi e sugli alienati» e operano conformemente alla "prevenzione e difesa sociale». L'analisi di «Quale giustizia» si focalizzò essenzialmente sulle tematiche di esclusione dei malati e di prevenzione dei sani nei confronti di coloro che risultano di pubblico scandalo. La legge in questione, sopravvissuta senza grossi cambiamenti durante il fascismo e la fase costituzionale, venne letta come uno strumento repressivo

\footnotetext{
${ }^{16}$ LIBERTINI, Raffaele, «Il manicomio non ha mai fine», in Quale giustizia, 29, 1974, p. 624. 17 «Manicomio a vita. Le situazioni giuridiche sospese», in Quale giustizia, 29, 1974, pp. 568580 .
} 
di controllo sociale e di salvaguardia della «morale piccolo-borghese», all'interno di un «sistema economico basato sul profitto [che] esclude le forze-lavoro improduttive» ${ }^{18}$.

Il testo della legge riporta che possano essere considerati «manicomi» tutti quegli «istituti, comunque denominati, nei quali vengono ricoverati alienati di qualunque genere»: non vi è quindi una definizione precisa dello spazio adibito agli infermi, perché in realtà è importante soprattutto che si tratti di un luogo idoneo a isolarli dalla società. D'altra parte, cosi come non è definito precisamente il manicomio, nel testo della legge non viene definita la natura della malattia mentale e su questo punto i redattori di «Quale giustizia» si concentrarono mostrando il circolo vizioso creatosi: «sono gli alienati che caratterizzano un luogo come manicomio; d'altra parte è il manicomio a caratterizzare come alienati $\mathrm{i}$ ricoverati in esso» ${ }^{19}$. Le uniche caratteristiche che la legge esplicita perché determinate persone possano essere ridotte in manicomio sono l'essere «pericolose a sé e agli altri» e il riuscire «di pubblico scandalo».

Proprio per semplificare l'isolamento di chi poteva turbare l'ordine pubblico, la procedura d'ammissione agli istituti era piuttosto rapida e regolata da certificati medici e ordinanze dei pretori. La stessa cosa non si poteva dire dell'eventuale «licenziamento dal manicomio», il cui procedimento era complesso e richiedeva tempi alquanto dilatati $^{20}$. Un altro aspetto importante regolato dalla legge del 1904 era il fatto che la vigilanza su manicomi e «alienati» fosse affidata al Ministro dell'Interno e ai prefetti: il personale di guardia non era quindi composto solo da infermieri, ma anche da guardie vere e proprie. Era inoltre ammessa la possibilità che l'infermo di mente potesse essere ricoverato in una casa di cura privata, equiparata negli artt. 1 e 2 agli istituti pubblici. Gli interventi di «Quale giustizia» mostrano che tale distinzione sia fondamentalmente di natura economica: chi aveva i mezzi per farlo preferiva ricorrere agli istituti privati, che però, dietro una parvenza di pulizia, efficienza e buon trattamento dei ricoverati, spesso non tenevano conto neanche delle poche misure di tutela nei confronti dei malati disposte dalla legge. I casi di abuso di autorità, di soprusi e maltrattamenti erano frequentemente riportati dalla stampa italiana, come vedremo meglio nel terzo capitolo.

Se gli ospedali psichiatrici rappresentavano uno strumento di controllo sociale, nei manicomi giudiziari «Quale giustizia» lesse una «doppia violenza istituzionale»,

18 AMBROSINI, Giangiulio, CECCARELLI PULITANÒ, Elisa, «L'esclusione manicomiale e la sua legge», in Quale giustizia, 17-18, 1972, p. 581.

19 Ibidem, p. 580.

${ }^{20}$ Legge 14 febbraio 1904, n. 36, Disposizioni sui manicomi e sugli alienati. Custodia e cura degli alienati, artt. 2 e 3. 
l'unione cioè dei sistemi coercitivi del carcere e del manicomio ${ }^{21}$. Nonostante il Codice penale definisse «incomunicabili» queste due istituzioni, dal momento che il carcere si doveva occupare delle persone imputabili «in grado di intendere e di volere» (quindi anche in grado di «emendarsi»), il manicomio invece delle persone non imputabili per «infermità mentali», sul piano effettuale non c'è differenza tra cura e repressione, tra custodia e carcerazione dal momento che, notano gli autori della rivista, «alla base della carcerazione manicomiale vi è una responsabilità oggettiva, la colpa di essere malato di mente o di essere considerato tale da altri»22. Questa confusione tra delinquenza e disadattamento portò a una «penosa e irrazionale promiscuità» nei manicomi giudiziari tra persone realmente malate e altre invece che vi erano ricoverate. Una lettera, inviata il 16 dicembre 1971 dal giudice di sorveglianza Alessandro Margara al Ministero di Grazia e Giustizia sulla situazione nel carcere giudiziario di Montelupo Fiorentino, riporta che molti dei presenti in fase di osservazione siano spesso soggetti «difficili», senza una caratterizzazione psichiatrica completa. Ciò comportava il sovraffollamento della struttura e impediva di svolgere terapie utili per i pochi internati realmente malati, mentre quelli che non lo erano venivano sottoposti in maniera controproducente allo stress dell'ambiente manicomiale 23 .

L'altra grossa questione affrontata nelle pagine di «Quale giustizia» fu proprio l'utilità ai fini terapeutici di molte delle pratiche adottate dal personale degli istituti manicomiali. L'utilizzo in molti casi del letto di contenzione, al quale venivano legati i malati più agiati o gli internati nei manicomi criminali, è un esempio di come discutibili sistemi di cura fossero tutt'uno con i mezzi di controllo fisico delle persone. L'ettrourtoterapia, l'insulinoterapia, e in molti casi anche psicofarmacoterapia vennero considerati metodi controproducenti o - nella migliore delle ipotesi - non utili ai fini della cura, sia dai rappresentanti di Magistratura democratica sia dagli psichiatri vicini a Basaglia, dal momento che fissavano il malato nel suo «ruolo passivo» impostogli dal sistema ${ }^{24}$.

In linea coi principi di rispetto della Costituzione, l'azione comune di «Quale giustizia» e di Psichiatria democratica evidenziò il fatto che i manicomi italiani fossero una delle facce di un vasto sistema coercitivo, attraverso l'individuazione «della natura di classe delle norme discriminanti, emarginanti, repressive» che regolavano questi

${ }^{21}$ CAPPELLI, Igino, «Il manicomio giudiziario», in Quale giustizia, 17-18, 1972, p. 620.

22 AMBROSINI, Giangiulio, CECCARELLI PULITANÒ, Elisa, «L'esclusione manicomiale e la sua legge», in Quale giustizia, 17-18, 1972, p. 584.

23 «Il manicomio giudiziario come doppia esclusione», in Quale giustizia, 17-18, 1972, pp. 62233.

${ }_{24}^{2}$ BASAGLIA, Franco (a cura di), op. cit., p. 146; «Sono matti: suicidiamoli», in Quale giustizia, 41-41, 1977, pp. 702-703. 
istituti ${ }^{25}$. Tutto ciò si evince anche dalle scelte lessicali: negli articoli della rivista termini come «internati», «custodia», «repressione» e «controllo» risultano molto più frequenti di «ricoverati» e «cura». Le difficoltà maggiori di questa riforma erano dovute a realtà strutturali, regolamentari e funzionali ormai consolidate da decenni di pratica, le quali rendevano inutili anche figure come il giudice di sorveglianza degli istituti psichiatrici giudiziari, il cui compito si limitata ad assicurare il rispetto minimo delle procedure di ammissione e uscita dei malati.

Messe in luce - in base alla lettura marxista della società - le cause strutturali (rapporti di produzione) e sovrastrutturali (norme e valori) che regolavano questo meccanismo di esclusione, ecco allora che il fine della battaglia dei medici e dei magistrati fu quello di mostrare anzitutto che queste pratiche fossero controproducenti e annichilenti per le persone in questione e, d'altra parte, che fosse possibile intraprendere un percorso terapeutico alternativo a quello repressivo in vigore, rendendo «volontaria» l'applicazione delle cure. La lotta, allargata poi all'intero sistema sanitario italiano, puntava a una riforma radicale dell'ordinamento.

\subsection{I rilievi di incostituzionalità della legislazione sui manicomi}

Il terreno su cui si concentrò maggiormente l'attività di Magistratura democratica perché si arrivasse alla chiusura degli ospedali psichiatrici fu quello della legittimità costituzionale: nel decennio delle riforme, la questione manicomiale ricoprì un ruolo importante e la Corte costituzionale fu chiamata a esprimersi sulla legislazione che regolava i processi ai malati di mente, i ricoveri e il funzionamento dei manicomi. In questo modo, l'appello ai valori fondativi del diritto italiano fornì un valido strumento per la sua riforma in chiave più democratica.

Una delle problematiche approfondite nelle pagine di «Quale giustizia» è quella delle situazioni giuridiche sospese in relazione a uno stato di malattia mentale, a partire dalla relazione del giudice di sorveglianza del Tribunale di Firenze Margara su alcuni ricoverati del manicomio giudiziario di Montelupo, i quali si trovavano di fatto in un «ergastolo di sicurezza» ${ }^{26}$. L'art. 148 del Codice penale prevedeva, in caso di infermità mentale sopravvenuta, la sospensione di un procedimento penale, della pena o delle misure detentive. Tutti questi casi comportavano il ricovero in manicomi giudiziari e spettava al giudice di sorveglianza decidere se far cessare questa sospensione, qualora il soggetto avesse recuperato le capacità di intendere e volere. Ciò che l'articolo della

25 «Il convegno nazionale di Psichiatria Democratica», in Quale giustizia, 30, 1974, p. 767.

26 «Manicomio a vita. Le situazioni giuridiche sospese», in Quale giustizia, 29, 1974, p. 568. 
rivista fa notare è che era impossibile auspicare un miglioramento delle condizioni psichiche dei sospesi a giudizio, visto l'ambiente carcerario in cui venivano posti: venivano a crearsi invece «situazioni di perdita della libertà personale praticamente perpetue», con casi frequenti di sospensione non giustificata 27.

Per questo motivo, si auspicava una scrupolosa perizia psichiatrica del soggetto, per comprendere se l'infermità fosse sopravvenuta al fatto o lo precedesse, nel qual caso l'imputato andava prosciolto. Per i ricoverati a pena sospesa invece, in primo luogo le critiche andavano contro le negligenze nelle perizie stesse, che facevano sì che molte persone venissero «dimenticate nei manicomi» ${ }^{28}$. D'altra parte venne messa in luce che, in base alla legge, il periodo di restrizione personale in manicomio era irrilevante ai fini dell'espiazione della pena. Secondo gli autori degli articoli di «Quale giustizia», ciò era l'ulteriore dimostrazione del carattere segregativo e punitivo del Codice penale: la questione era che andavano salvaguardati la consapevolezza e la partecipazione cosciente dei soggetti rispettivamente alla pena o al processo, non tanto la guarigione dall'infermità psichica, pressoché impossibile viste le condizioni disastrose dei manicomi giudiziari ${ }^{29}$.

La questione della legittimità costituzionale dell'art. 148 del Codice penale e dell'art. 88 del Codice di procedura penale venne sollevata a partire dall'art. $24,2^{\circ}$ Comma della Costituzione, che nel diritto alla difesa prevedeva la partecipazione dell'interessato e la sua assistenza per mezzo di un difensore. Fu sostenuta l'illegittimità del regime di sospensione dell'esecuzione della pena per infermità sopravvenuta e l'incostituzionalità del fatto che il periodo trascorso in manicomi giudiziario con pena sospesa non venisse sottratto alla pena da espiare. Il principio ispiratore della norma non poteva essere la correzione dell'imputato attraverso l'afflizione. Dopo un iter piuttosto lungo, la Corte costituzionale si espresse nel giugno 1975, dichiarando illegittimo l'art. 148

nella parte in cui prevede che il giudice, nel disporre il ricovero in manicomio giudiziario del condannato caduto in stato d'infermità psichica durante l'esecuzione di pena restrittiva della libertà personale, ordini che la pena medesima sia sospesa. Ha dichiarato del pari l'incostituzionalità dell'art. 148 nella parte in cui prevede che il giudice ordini la sospensione della pena anche nel caso in cui il condannato sia

\footnotetext{
${ }^{27}$ Ibidem, p. 570.

${ }^{28}$ Esempi di queste "dimenticanze" sono G. Angioni, B. Vangiu, i quali erano innocenti o infermi prima di commettere il delitto. Caso emblematico è quello di Andrea Pellerano che, pur dovendo scontare solo tre anni di carcere per furto, ritenuto insano di mente ne passò venti in un manicomio giudiziario; cfr. CARBONE, Fabrizio, «Doveva scontare 3 anni per furto. Ne passò venti in un manicomio», La Stampa, 29 aprile 1975, p. 11.

29 LIBERTINI, Raffaele, «Il manicomio non ha mai fine», in Quale giustizia, 29, 1974, p. 621.
} 
ricoverato in una casa di cura e di custodia ovvero in un manicomio comune (ospedale psichiatrico) ${ }^{30}$.

Tuttavia fu dichiarata inammissibile la questione di incostituzionalità in riferimento all'art. 24 della Costituzione.

La reticenza da parte della Corte costituzionale a una riforma radicale del sistema giudiziario in riferimento ai manicomi venne letta dagli autori della rivista come una «scelta politica»: non vi era intenzione di compromettere l'impianto legislativo della legge del 1904 che faceva perno sul concetto di «pericolosità sociale» degli infermi di mente. A questo proposito venne riportata la richiesta da parte giudice Cerminara della Pretura di Roma di verifica della costituzionalità dei primi due articoli della legge, in base ai quali un pretore poteva mandare provvisoriamente in manicomio un malato se c'era - come si è detto - una richiesta da parte di parenti o tutori, un certificato di un «medico esercente» e un atto di notorietà, il tutto nell'interesse della società e del malato. Il giudice romano insisteva che queste misure contrastassero con l'art. 32 della Costituzione in materia di trattamenti sanitari, per cui «la legge non può in nessun caso violare i limiti imposti dal rispetto della persona umana», sebbene venga ammesso in alcuni casi l'obbligo di un determinato trattamento per disposizione di legge. Il punto cruciale era però che i malati di mente non venivano considerati «persone», bensì soggetti pericolosi per la società, che provvede pertanto alla loro esclusione e custodia in luoghi separati ${ }^{31}$.

\section{Le cronache giudiziarie}

L'analisi e la critica della società italiana condotte dai giuristi che scrivevano su «Quale giustizia» erano integrate con sentenze di vario argomento che venivano riportate per intero. È questa probabilmente una delle caratteristiche più interessanti della rivista, in grado di arricchire il dibattito con esempi concreti di applicazione della giustizia. Si trattava spesso di casi che trovavano spazio anche nelle cronache dei quotidiani e, per quanto riguarda il tema della salute mentale, in gran maggioranza sono riportati per mostrare le forme di coercizione presenti nella legislazione italiana, tra cui spicca l'istituzione manicomiale.

${ }^{30}$ Corte costituzionale, 19 giugno 1975, n. 146.

${ }^{31}$ Cfr. «La legge sui manicomi di nuovo sotto accusa», in Quale giustizia, 36, 1975, pp. 746-48; BASAGLIA, Franco (a cura di), op. cit., pp. 122-123. 
Una delle sentenze citate è quella della Pretura di Parma del 21 aprile 1972, riguardante la denuncia esposta dal dottor Euplio Mastrangelo contro il medico di guardia dell'Ospedale psichiatrico il Colorno, il dottor Franco Rotelli. Questi avrebbe rifiutato «per ragioni di ordine specialistico-sanitario e per ragioni di ordine burocratiche amministrative (sic.) in quanto proveniente da altra provincia»32 il ricovero di Tiziano Bigi, persona affetta da «etilismo cronico»; costui si era precedentemente rivolto al dottor Mastrangelo, il quale aveva riconosciuto i sintomi di «deterioramento psichico» e aveva chiesto all'autorità di pubblica sicurezza il ricovero d'urgenza in via provvisoria, approvata dal Questore. Con questo esempio si vuole mettere in luce il potere della pubblica amministrazione e dell'atto d'ordinanza: senza aver fatto ricorso agli strumenti di interpretazione dell'ordinanza e senza aver effettuato una visita più accurata del signor Bigi, ciò che risulta è la messa in discussione dell' «Autorità» dello Stato. Il rifiuto del dottor Rotelli era perciò attribuito a una mancanza di rispetto delle istituzioni33. D'altra parte, il caso è un'ulteriore dimostrazione che la semplicità con la quale si poteva entrare in manicomio - seppur in via provvisoria - non fosse controbilanciata da una rapida dismissione, qualora si fosse provveduto a una perizia psichiatrica più accurata.

Accanto alla critica del concetto di autorità, le pagine di «Quale giustizia» riportano anche due episodi drammatici di morte avvenuti nei manicomi. Era piuttosto raro che la responsabilità dei fatti ricadesse sugli infermieri di reparto o sui medici stessi ed erano poche le cronache giudiziarie che trattavano casi di questo tipo: tuttavia quelle poche condanne registrate sono utili per comprendere quali fossero che condizioni all'interno degli ospedali psichiatrici.

Il primo di questi due casi riguarda la condanna del prof. Benigno Di Tullio, titolare della Casa di Salute per Malattie Nervose Parco delle Rose: si trattava di una cosiddetta «clinica aperta» privata, dove potevano essere ricoverati solo malati «non pericolosi», ai quali era concessa maggiore libertà, e dove erano assenti rigide misure di sicurezza e personale diverso dagli infermieri. Il 12 ottobre 1973 vi fu ricoverato tale B. A., ritenuto sofferente di schizofrenia catatonica, non pericolosa per sé. Egli però il 14 ottobre, elusa la sorveglianza, riuscì ad allontanarsi dalla clinica e venne ritrovato impiccato il giorno dopo. Questo triste caso, attribuito inizialmente a un difetto di sorveglianza, viene riportato nella rivista in quanto esempio di come le cliniche private tendessero a eludere la legislazione corrente: la casa di cura in questione - viene detto - «era formalmente una clinica aperta ma operava, sostanzialmente, come clinica aperta e

32 «Il manicomio facile», in Quale giustizia, 17-18, 1972, p. 469.

33 Ibidem, p. 470. 
come clinica chiusa»34, dal momento che era divisa in due reparti, uno dei quali era dotato di reti metalliche, finestre con inferriate e porte di sicurezza. In base a ciò, era equiparabile ai manicomi chiusi pubblici, ai sensi dell'artt. 1 e sgg. 1. 14.2.1904 n. 36 e artt. 1, 2, 3, 4 e segg. del R.D. 16.8.1909 n. 615, cosa non rispettata dalla Casa Parco delle Rose. Non solo: l'art. 2 della legge 18.3.1968 n. 431 prevedeva un «rapporto di un infermiere per ogni tre posti-letto», anch'esso eluso essendo presenti nella clinica solo due infermieri per un totale di 45 ricoverati al momento del fatto.

Oltre al non rispetto del regolamento, la questione di fondo è che non si sia tenuto conto del fatto che il paziente soffrisse in realtà di schizofrenia paranoica: nella sua cartella clinica erano registrati infatti casi precedenti di tentato suicidio, di allucinazioni e di istinti omicidi. Durante il processo, il pubblico ministero cercò di sciogliere il nesso tra azione-omissione colposa del dottor Di Tullio e il fatto delittuoso, sostenendo che il malato avesse scelto coscientemente il suicidio. In realtà, la condanna fu di aver accettato nella propria clinica non idonea un malato che presentava gravi sintomi, dei quali però non si era tenuto conto. Questo caso mostra, secondo i redattori della rivista, quanto dietro a un'apparenza di maggior pulizia ed efficienza delle cliniche private vi fossero in realtà interessi economici, non terapeutici: tale problema non era quindi caratteristica esclusiva delle strutture pubbliche, ma coinvolgeva l'istituzione manicomiale nel suo complesso 35 .

Uno dei casi tristemente più noti di violenza fu quello avvenuto nel manicomio giudiziario femminile di Pozzuoli, dove la signora Antonia Bernardini, arrestata per oltraggio e lesioni a pubblico ufficiale e sofferente di ripetuti esaurimenti nervosi, era ricoverata in attesa di giudizio. Pur essendo accusata di un reato da quattro o cinque mesi con la condizionale, la Bernardini rimase a Pozzuoli più di un anno, finché nella notte del 27 dicembre 1974, nonostante fosse legata al letto di contenzione, riuscì a darsi fuoco; morì il 31 dicembre in seguito alle ustioni riportate. Si trattò di una notizia, resa pubblica solo il 4 gennaio 1975 , che ebbe una vasta risonanza mediatica ${ }^{36}$, fino alla sentenza del 17 giugno 1977 con cui il Tribunale di Napoli condannò per omicidio colposo, abuso di autorità e di falso ideologico in atto pubblico i responsabili: quattro anni di carcere furono dati al dottor Francesco Corrado (direttore dell'ospedale), quattro e mezzo al dottor Giuseppe Tempone (direttore di reparto), un anno e mezzo alla suora responsabile del personale e a tre vigilatrici.

34 «Sono matti: suicidiamoli», in Quale giustizia, 41-42, 1977, p. 699.

35 Ibidem, pp. 696-700.

${ }^{36}$ Cfr. «Bloccata nella camicia di forza si libera e si dà fuoco: è morta», La Stampa, 5 gennaio 1975, p. 9; PUNTILLO, Eleonora, «Senza soccorsi muore bruciata legata al letto di contenzione», in l'Unità, 5 gennaio 1975, p. 5. 
Riguardo questo caso, il dibattito di «Quale giustizia» è incentrato sulla liceità e utilità come strumento terapeutico del letto di contenzione e delle altre forme coercitive che possono incidere violentemente sulla personalità del malato. Il ricorso a esse era ancora permesso nelle strutture, nonostante fosse in qualche modo limitato o regolato da norme, soprattutto in ospedali come quello di Pozzuoli, che all'epoca poteva vantare poche carenze dal punto di vista della manutenzione e del personale. Qualora però si fosse dimostrato che l'uso di mezzi coercitivi fosse stato fatto «per motivi utilitaristici gravemente censurabili e con effetti terapeutici addirittura controproducenti» 37 , a quel punto non erano più considerate pratiche lecite. Dalle indagini si scoprì che le pazienti ospitate a Pozzuoli erano frequentemente legate al letto nelle ore notturne, solo per garantire al personale di sorveglianza una maggior tranquillità e non per scopi terapeutici. Inoltre, contrariamente a quanto stabilito dal regolamento, i provvedimenti coercitivi erano disposti in maniera informale, senza indicazioni sulla cartella clinica e senza tener conto dell'effetto deleterio che essi avevano su molte delle internate, tra cui la signora Bernardini. Questo era stato fatto anche nei giorni precedenti al fatto: prima di andare in vacanza, il dottor Tempone aveva firmato in bianco il registro delle contenzioni, cosicché le vigilatrici potessero regolarsi come meglio credevano nei giorni della sua assenza, risparmiando la fatica di contattarlo per chiedergli il via libera. Lo stesso direttore del centro, il dottor Corrado, non andò a sostituire Tempone durante la sua assenza. Per questo atto i due «debbono rispondere del reato continuato di cui all'art. 608 c.p.; della fattispecie di cui all'art. 586 c.p. e il secondo, altresì, del delitto di falso ideologico in atto pubblico» 38 .

All'interno di questa vicenda è possibile ritrovare i punti principali sui quali si batté «Quale giustizia». La signora Bernardini soffriva di una patologia che non era mai stata analizzata accuratamente: tuttavia era considerata persona indesiderabile, in quanto mentalmente instabile ed economicamente non produttiva. Il fatto che si fosse recata più volte - di sua spontanea volontà - al manicomio civile di Roma per essere curata quando era in preda a crisi depressive, la inserì nel meccanismo di esclusione sociale che la etichettava come «malata di mente». Accusata poi di un delitto non grave, fu dimenticata in un manicomio giudiziario che, dietro la facciata della pulizia e della professionalità, nascondeva pratiche discutibili e controproducenti a livello terapeutico. Alla luce di tutti questi aspetti, la Bernardini rappresenta appieno una vittima della

37 «Sono matti: suicidiamoli», in Quale giustizia, 41-42, 1977, p. 703.

${ }^{8}$ Ibidem, p. 704; cfr. PUNTILLO, Eleonora, «Condannati per Antonia Bernardini bruciata viva nel carcere-manicomio», in l'Unità, 18 giugno 1977, p. 5 . 
«doppia esclusione»39 veicolata dagli ospedali psichiatrici (specie quelli giudiziari), tematica centrale della battaglia condotta da Psichiatria democratica contro l'istituzione manicomiale.

\section{Conclusioni. La legge 1978, n. 180}

I collaboratori di «Quale giustizia» si rendevano conto che la riforma del sistema sanitario italiano, della quale il Parlamento iniziò a occuparsi nel 1977, dovesse in qualche modo rivoluzionare anche il modo in cui veniva affrontata la malattia mentale. Era necessario però coinvolgere un sistema più ampio che comprendeva manicomi giudiziari e carceri, tutti parte di un meccanismo di esclusione di chi era considerato pericoloso e - soprattutto - irrecuperabile, meccanismo su cui si erano fondate società e istituzioni.

Nel corso del «decennio riformista», l'esigenza di una riforma della normativa psichiatrica andò di pari passo con l'allargamento della democrazia che la società civile portava avanti in forme fino ad allora inedite o profondamente mutate se già esistevano: le organizzazioni sindacali, le proteste di piazza, la mobilitazione dell'opinione pubblica attraverso riviste o trasmissioni radiofoniche, i referendum. Tutti questi stimoli «dal basso» contribuirono ad accelerare la spinta alle riforme attuate dai governi di coalizione tra democristiani e socialisti, nella prima metà degli anni Settanta, e poi dai due governi di unità nazionale. D’altra parte però, il ruolo delle istituzioni fu tutt'altro che passivo: esse accolsero al loro interno alcune delle istanze provenienti dalla società civile più progressista, ma ciò comportò in molti casi l'abbandono delle posizioni più radicali quali poteva avere - nel nostro caso - il movimento anti-istituzionale basagliano. In questo modo il ricorso al diritto e ai principi costituzionali come strumento di contestazione operato da Magistratura democratica può aver avuto come conseguenza indiretta la legittimazione di quello Stato contro il quale si protestava ${ }^{40}$. Nonostante la messa da parte di molti obiettivi che questi movimenti si erano dati, essi riuscirono nel caso della salute mentale a porre l'accento sul fatto che essa fosse in primo luogo una questione sociale, non solo biologica. Ciò che la rivista «Quale giustizia» e Psichiatria democratica cercarono di fare fu quindi porsi «come punto di riferimento tecnico per le forze politiche

39 Cfr. nota 23.

40 È questa una delle problematiche più interessanti messe in luce ne Le armi del diritto, nel momento in cui si parla di «legittimazione involontaria» e di «relativa eufemizzazione dei conflitti» qualora la contestazione si serva del diritto: cfr. ISRAËL, Liora, op. cit., pp. 79-84. 
progressiste» 41 indirizzate alla chiusura dei manicomi, interpretati come simbolo lampante di controllo ed esclusione.

Dopo che la legge ponte del 1968 ebbe modificato in parte le condizioni interne ai manicomi, nell'aprile 1977 la Camera dei deputati diede il via alla discussione sul disegno di legge «Istituzione del servizio sanitario» (la futura legge 1978, n. 833), all'interno del quale doveva essere trattata anche l'assistenza psichiatrica. Il ruolo di mediatore tra le varie sollecitazioni fu svolto dal deputato democristiano e psichiatria Bruno Orsini: se da un lato si era d'accordo sull'abrogazione della legge manicomiale del 1904 e 1909, dall'altro l'ammissibilità e la regolamentazione del ricovero non volontario fu fonte di dibattito, per le implicazioni costituzionali, politiche e sociali che esso aveva. La stesura legge quadro n. 180, inoltre, rispose alla necessità di uscire da una situazione difficile. Entro l'11 maggio 1978 il disegno di legge avrebbe dovuto essere approvato, altrimenti si sarebbe proceduto a un referendum sull'abolizione della normativa precedente (proposto dai radicali), il cui esito però non era scontato. Inoltre erano quelli i mesi in cui l'Italia visse la vicenda del sequestro del presidente della Democrazia cristiana Aldo Moro.

In questo clima, il disegno di legge portato avanti da Orsini sulla riforma dell'assistenza psichiatrica fu presentato, discusso e approvato in tempi record da Camera e Senato: il 10 maggio 1978 la legge n. 180 «Accertamenti e trattamenti sanitari volontari e obbligatori» venne firmata dal capo dello Stato Giovanni Leone, dal guardasigilli Paolo Bonifacio e dal ministro della Sanità Tina Anselmi. Venne così attuata una rivoluzione nella psichiatria italiana: i manicomi sarebbero stati chiusi. Tuttavia la 180 non poteva essere la «legge Basaglia», come spesso è definita: non era nemmeno una legge a se stante, ma parte del sistema sanitario nazionale. Lo stesso Basaglia il giorno prima della ratifica sottolineò il fatto che si trattasse certamente di un compromesso politico, per cui era consigliabile evitare facili euforie: «non si deve credere di aver trovato la panacea a tutti i problemi del malato di mente con il suo inserimento negli ospedali tradizionali» 42 .

Nonostante i risultati non fossero all'altezza delle aspettative di coloro che, nel corso degli anni, si erano battuti per la chiusura dei manicomi, la legge n. 180 rappresentò un tappa fondamentale del processo di democratizzazione della società italiana e di riconoscimento della dignità di persona e dei diritti civili e politici anche di coloro che potevano essere sottoposti a trattamenti sanitari obbligatori. Questo saggio breve ha cercato di mostrare quanto, nel raggiungimento di questo traguardo, l'uso del diritto

${ }^{41}$ BABINI, Valeria P., op. cit., p. 285.

$4^{2}$ GILIBERTO, Franco, «Che dice Basaglia», in La Stampa, 12 maggio 1978, p. 12. 
come arma per mobilitare l'opinione pubblica e spingere alle riforme abbia giocato un ruolo centrale. Tuttavia, la chiusura dell'esperienza di «Quale giustizia» col numero di giugno 1979, pressoché in concomitanza col tramonto delle grandi riforme del decennio, lascia aperta la domanda se il ricorso al diritto con obiettivi politici o sociali possa portare a risultati concreti per una vera democratizzazione della società anche al giorno d'oggi, o se invece il formalismo e la sovrapposizione di norme e regolamenti lo rendano al contrario uno mezzo per disinnescare tale spinta alle riforme ${ }^{43}$. Al di là di questi interrogativi, il contributo di Magistratura democratica e dalla sua rivista fu senz'altro importante per superare la definizione che la legge del 1904 dava dei malati di mente come persone «pericolose a sé o agli altri [e] di pubblico scandalo» 44 .

43 ISRAËL, Liora, op. cit., pp. 108-109.

44 Legge 14 febbraio 1904, n. 36, art. 1. 


\section{* L'autore}

Francesco Mantovani ha studiato Storia presso l'Università di Bologna, dove ha conseguito la Laurea triennale nel 2013 con una tesi sul nuovo ordine mediterraneo fascista. Iscritto a Scienze storiche nello stesso ateneo, partecipa attualmente al secondo anno del Corso di Laurea Magistrale integrato italo-tedesco in Scienze storiche (BiBoG) presso l'Universität Bielefeld. URL: < http://www.studistorici.com/progett/autori/\#Mantovani >

\section{Per citare questo articolo:}

MANTOVANI, Francesco, «Esclusione sociale e violenza istituzionale II tema della salute mentale in "Quale giustizia"», Diacronie. Studi di Storia Contemporanea : II diritto militante, 29/12/2014,

URL:< http://www.studistorici.com/2014/12/29/mantovani_numero_20/ >

Diacronie Studi di Storia Contemporanea $\beta$ www.diacronie.it

Risorsa digitale indipendente a carattere storiografico. Uscita trimestrale.

redazione.diacronie@hotmail.it

Comitato di redazione: Jacopo Bassi - Luca Bufarale - Elisa Grandi - Deborah Paci - Fausto Pietrancosta - Matteo Tomasoni - Luca Zuccolo 